\title{
Invisible waveguides on metal plates for plasmonic analogs of electromagnetic wormholes
}

\author{
Muamer Kadic, Guillaume Dupont, Stefan Enoch, and Sebastien Guenneau \\ Aix-Marseille Université, CNRS, Centrale Marseille, Institut Fresnel, Campus universitaire de Saint-Jérôme, 13013 Marseille, France
}

(Received 20 April 2014; revised manuscript received 6 June 2014; published 10 October 2014)

\begin{abstract}
We introduce two types of toroidal metamaterials which are invisible to surface plasmon polaritons (SPPs) propagating on a metal surface. The former is a toroidal handlebody bridging remote holes on the metal surface: It works as a kind of plasmonic counterpart of electromagnetic wormholes. The latter is a toroidal ring lying on the metal surface: This bridges two disconnected metal surfaces, i.e., it connects a thin metal cylinder to a flat metal surface with a hole. Full-wave numerical simulations demonstrate that an electromagnetic field propagating inside these metamaterials does not disturb the propagation of SPPs at the metal surface. A multilayered design of these devices is proposed, based on effective medium theory for a set of reduced parameters: The former plasmonic analog of the electromagnetic wormhole requires homogeneous isotropic magnetic layers, while the latter merely requires dielectric layers.
\end{abstract}

DOI: 10.1103/PhysRevA.90.043812 PACS number(s): 42.70.-a, 41.20.-q, 03.50.De, 73.20.Mf

\section{INTRODUCTION}

Eight years ago, two groups of physicists [1,2] unveiled independent paths towards electromagnetic invisibility. The transformational optics proposal by Pendry et al. leads to singular tensors on the frontier of the invisibility region $[3,4]$ that require an extreme electromagnetic response achieved upon resonance of split ring resonators [5]. Various extensions including the blowup of a segment instead of a point [6,7] and the stereographic projection of a virtual hypersphere in a four-dimensional space [8] have been studied. The conformal optics proposal by Leonhardt's grouping [2] leads to spatially varying, but bounded, scalar permittivity and permeability. However, the mathematical tools of complex analysis thus far constrain the invisibility design to two dimensions. Some recent advances in quasiconformal optics [9-12] also found some applications in the control of surface plasmon polaritons (SPPs): Transformational plasmonics [1320]. Harnessing SPPs in order to deliver coupling between surface electrons on a structured metallic plate and incident light is a core topic in plasmonics [21-24], and plasmonic resonances underpins invisibility relying upon devices such as out-of-phase polarizability shells with low refractive index [25], core-shell anomalous resonances [26], or concentric rings of point scatterers [27]. However, the field of transformational plasmonics has a broader range of applicability as it is fueled by analogies with Einstein's general relativity, such as electromagnetic wave propagation in inhomogeneous media and particle or light motion in gravitational potentials. For example, the plasmonic Eaton lens proposed by Zhang's team [19] is reminiscent of an optical black hole [28-30], which can trap and absorb electromagnetic waves coming from all directions. In the present article, we adapt the design of transformation based wormholes to the area of SPPs. In physics and fiction, a wormhole is portrayed as a shortcut

Published by the American Physical Society under the terms of the Creative Commons Attribution 3.0 License. Further distribution of this work must maintain attribution to the author(s) and the published article's title, journal citation, and DOI. through space-time. Building upon the recent proposal of electromagnetic wormholes by Greenleaf et al. [7], it is enough to consider it as a topological feature of space. Our main contribution is an explicit design of a toroidal handlebody to control SPPs propagating at a metal surface with two holes: The main ingredient in the recipe of our plasmonic analog of the electromagnetic wormhole is to blow up a curve, rather than a point as is used in a typical three-dimensional invisibility cloak [31]. We further numerically demonstrate the validity of our theoretical approach with three-dimensional finite element computations for SPPs propagating in a toroidal heterogeneous anisotropic wormhole (we later abuse the word wormhole to refer to our toroidal cloak). We finally derive some reduced set of parameters allowing for the design of a multilayered toroidal tunnel consisting of an alternation of isotropic homogeneous layers approximating the ideal cloaking device in the homogenization limit. This brings our plasmonic analogs of electromagnetic wormholes a step closer to an experimental setup. Potential applications in plasmonics range from invisible plasmonic waveguides (which could be useful in making measurements of electromagnetic fields without disturbing them, or as new types of endoscopes in medical applications), to hard discs for optical computers (the latter requires a tilted version of the wormhole which lies on a metal surface, which is also discussed). Other applications can be also envisaged thanks to the unprecedented control of SPPs through transformational plasmonics.

\section{DESCRIPTION OF TOROIDAL HANDLEBODIES ON METAL PLATES}

In this article, we introduce two types of plasmonic analogs of electromagnetic wormholes. We start by describing the mathematical construction of a magnetic SPP analog to the electromagnetic wormhole, which involves an electromagnetic toroidal cloak thereafter called handlebody and two holes on a metal plate. Such a wormhole can be implemented for electromagnetic fields by deriving the required tensors of permittivity and permeability for a toroidal region of $\mathbb{R}^{3}$ (the invisible tunnel) connecting two regions of a metal surface, using the tools of transformational plasmonics. 


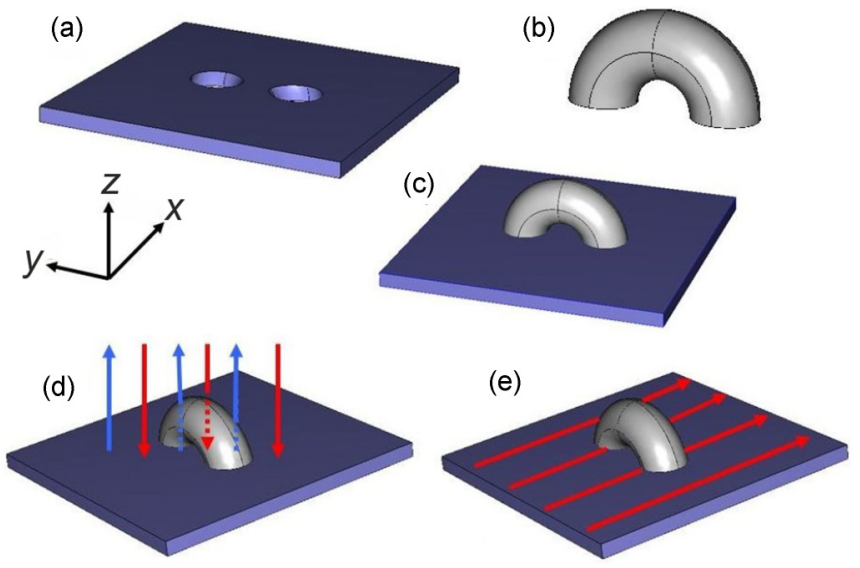

FIG. 1. (Color online) Main ingredients of plasmonic analog of electromagnetic wormhole I: (a) diagrammatic view of the metal plate with two remote holes, $M=\mathbb{R}^{2} \backslash\left(D_{1} \cup D_{2}\right)$, and (b) the toroidal handlebody $T=\partial M \times[0, L]$ bridging the holes; (c) these form a plasmonic analog of electromagnetic wormhole $W=M \cup T$; (d) diagrammatic view of streamlines for a normally incident plane wave on the holey metal plate with handlebody $W=M \cup T$ and (e) for a surface plasmon polariton (SPP) propagating on the holey plate $M$ along the toroidal axis, i.e., $x$ direction. The handlebody $T$ is invisible to any electromagnetic field (including SPPs).

The main ingredients of our wormhole construction are as follows: We start by making two identical holes in a metal plate [see Fig. 1(a)], for instance, two discs $D_{1}$ and $D_{2}$ separated by some positive distance on a plane. We denote by $M$ the region so obtained: $M=\mathbb{R}^{2} \backslash\left(D_{1} \cup D_{2}\right)$. Topologically, $M$ is a two-dimensional manifold with a boundary, the boundary of $M$ being $\partial M=\partial D_{1} \cup \partial D_{2}$. We note that $\partial M$ is the disjoint union of two discs on the plane.

The second component of the SPP analog to the electromagnetic wormhole $W$ is a curved toroidal cylinder [see Fig. 1(b)], $T=\partial M \times[0 ; L]$, where $L$ denotes the arc length which connects points of circle $\partial D_{1}$ to points of circle $\partial D_{2}$. As the boundaries of $M$ and $T$ are topologically the same ( $\left.\partial M=\partial T=\partial D_{1} \cup \partial D_{2}\right)$, we can glue these boundaries together. The resulting domain $W$ no longer lies on the metal surface $\mathbb{R}^{2}$, but rather has the topology of Euclidian space $\mathbb{R}^{3}$ with a three-dimensional handle attached to it, see Fig. 1(c). $\mathrm{W}$ is a two-dimensional space with a special topology which is embedded in the Euclidean physical three-dimensional space. We show in Fig. 1(d) a diagrammatic view of streamlines of a plane wave incident upon $\mathrm{W}$ (the ringlike handlebody is invisible; hence $\mathrm{W}$ reflects light like a flat metal surface) and ray trajectory of SPP propagating on $\mathrm{W}$ (there is no perturbation of the SPP trajectory induced by the holes and the ringlike handlebody), see Fig. 1(e).

Regarding the construction of the dielectric SPP analog to the electromagnetic wormhole, see Fig. 2, the previous construction repeats mutatis mutandis with the noticeable difference that the manifold $M$ should be replaced by a manifold $M_{1}$ with a single hole: $M_{1}=\mathbb{R}^{2} \backslash D_{1}$. Moreover, the disk $D_{2}$ is now a piece of metal located inside the hole $D_{1}$ : $D_{2} \subset D_{1}$, see Fig. 2(a). We fill in the hole with a toroidal cloak, see Fig. 2(b), which results in the dielectric type of SPP analog of the electromagnetic wormhole, see Fig. 2(c). We
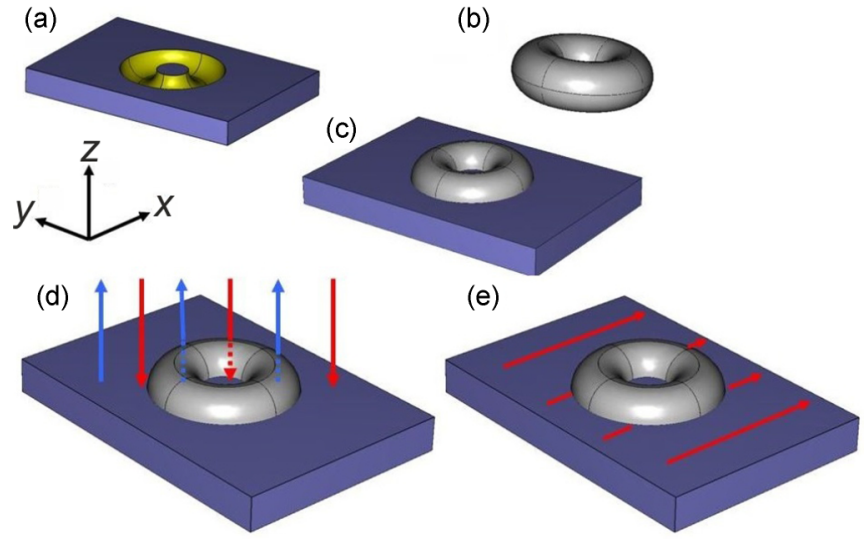

FIG. 2. (Color online) Main ingredients of plasmonic analog of electromagnetic wormhole II: (a) diagrammatic view of the metal plate with a large hole (manifold $M_{1}=\mathbb{R}^{2} \backslash D_{1}$ ) with a disconnected circular plate in its center (manifold $D_{2}$ ) (b) with the gold region depicting the region to be occupied by the toroidal handlebody $T_{1}$. (c) This results in a single manifold $W_{1}=M_{1} \cup T_{1}$; (d) diagrammatic view of streamlines for a normally incident plane wave on the holey metal plate $M_{1}$ with toroidal handlebody $T_{1}$ and (e) for a SPP propagating along the $x$ direction, i.e., orthogonal to the toroidal axis ( $z$ direction). Note that SPPs do propagate inside the center disk (this circular plate is glued to the infinite holey plate).

show in Fig. 2(d) a diagrammatic view of streamlines of a plane wave incident upon $W_{1}$ (the handlebody is invisible; hence $W_{1}$ reflects light like a flat metal surface) and ray trajectory of SPP propagating on $W_{1}$ (there is no perturbation of the SPP trajectory by the holes and the handlebody), see Fig. 2(e).

\section{TRANSFORMATION PLASMONICS FOR THE DESIGN OF PLASMONIC ANALOGS OF ELECTROMAGNETIC WORMHOLES}

We now wish to apply tools of transformational plasmonics to design a device in $\mathbb{R}^{3}$ which controls the propagation of SPPs in the same way as the presence of the handle $T$ in the wormhole manifold $W$. On $W$ we shall use the Riemannian metric that is the Euclidian metric on $M$ and the product metric on $T$. We emphasize that we are not actually tearing and gluing plasmonic space, but instead prescribing a metamaterial which makes the SPPs propagating on the metal plate (see Figs. 3 and 4) behave as if they were propagating on the wormhole manifold $W$. In other words, adopting the viewpoint of a SPP, it appears that the topology of plasmonic space has been changed.

We first consider a SPP propagating in the positive $x$ direction at the interface $z=0$ between a metal surface $(z<0)$ and air $(z>0)$, see Figs. 3 and 4 . If we choose the magnetic field $\mathbf{H}$ as the unknown, it takes the following form:

$$
\begin{aligned}
& \mathbf{H}_{2}=\left(0, H_{y_{2}}, 0\right) \exp \left\{i\left(k_{x 2} x-\omega t\right)-k_{z 2} z\right\}, z>0, \\
& \mathbf{H}_{1}=\left(0, H_{y_{1}}, 0\right) \exp \left\{i\left(k_{x 1} x-\omega t\right)+k_{z 1} z\right\}, z<0,
\end{aligned}
$$

with $\operatorname{Re}\left(k_{z 1}\right)$ and $\operatorname{Re}\left(k_{z 2}\right)$ strictly positive in order to maintain evanescent fields above and below the interface $z=0$. The amplitude of the incident field has a Gaussian profile $H_{y_{i}}=$ $e^{-\frac{y^{2}}{2 \delta^{2}}}$ with $\delta=3 \lambda$. 
(a)

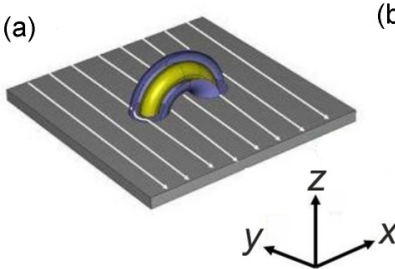

(b)
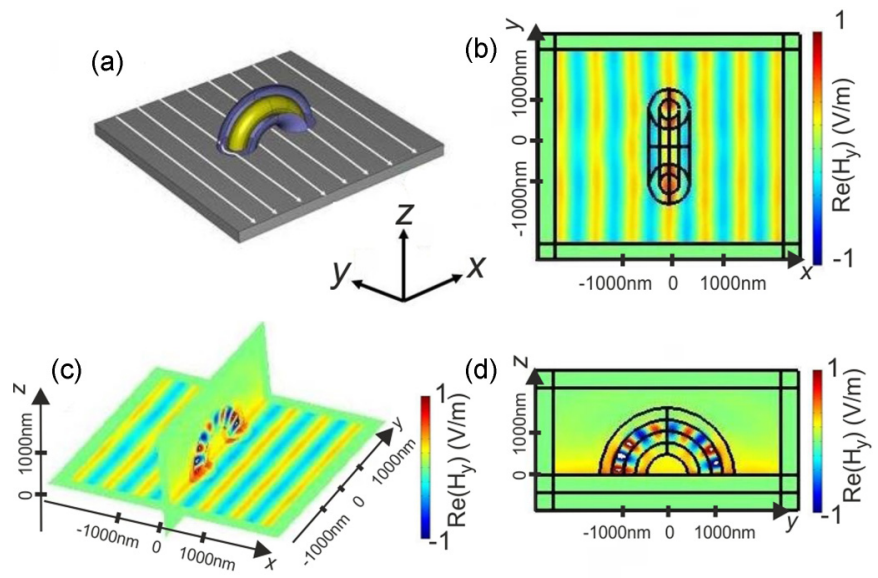

FIG. 3. (Color online) (a) Principle of the undetectable tunnel bridging two distant regions on a metal surface. The electromagnetic waveguide is shown in yellow and the coating in blue. It is coated with a transformed dielectric plasmonic analog of the electromagnetic wormhole with reduced parameters: in principle, the handlebody is acting as a waveguide for electromagnetic waves launched from the metal dielectric interface where we set $\mathbf{H}=\left(H_{x 2}, 0,0\right) \exp (-i \omega t)$ with $H_{x 2}=\exp (-i \sqrt{2} z$ ). (b) Two-dimensional plot (view from above) of the real part of the magnetic field. (c) Three-dimensional plot validating the guiding and invisibility properties. (d) Twodimensional plot of the real part of the magnetic field in the vertical plane showing the inner structure of the wormhole with a dielectric in the middle region which is surrounded by two regions of transformed medium. Note that the interfaces between the regions consist of a thin, conducting layer of thickness $70 \mathrm{~nm}$. These computations are for a wavelength of 700 for two remote circular holes of diameter $2 a=533$ with a center-to-center spacing of $2 R=1000 \mathrm{~nm}$.

\section{Plasmonics at a metal plate-wormhole interface}

Let us now consider two holes in the metal interface. It is clear that the propagation of the SPP is affected by

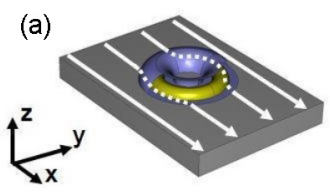

(c)

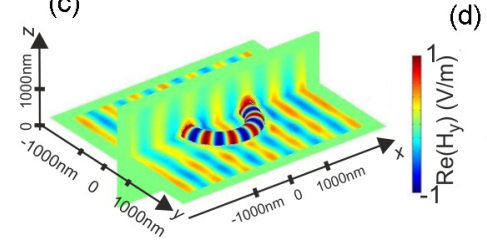

(b)

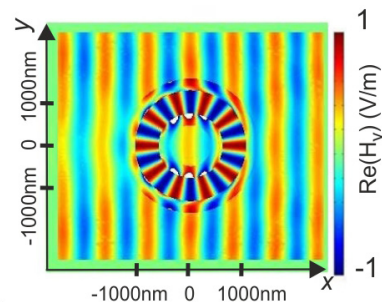

(d)

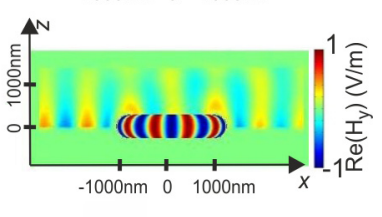

FIG. 4. (Color online) (a) Principle of the undetectable toroidal ring bridging two disconnected regions, i.e., a metal cylinder and a metal surface. The electromagnetic waveguide is shown in yellow and the coating in blue. Ray trajectories are drawn for illustrative purpose only. Full wave simulations validate the theoretical proposal: (b) View from top. (c) Three-dimensional (3D) plot. (d) Side view. Here, all plots are for the real part of the magnetic field and we set $\mathbf{H}=\left(0,0, H_{z 2}\right) \exp (-i \omega t)$ with $H_{x 2}=\exp (-i \sqrt{2} z)$ on the waveguide cross section in the vertical plane $y=0$. their presence, as illustrated in Fig. 6. Our aim is to design an invisible handlebody through geometric surgery which will bridge the two holes at the metal surface as shown in Fig. 1. For simplicity, we construct a device that has rotational symmetry about a line in $\mathbb{R}^{3}$, and moreover we assume that the radii of $D_{1}$ and $D_{2}$ are equal. We use toroidal coordinates $(r, u, v)$ corresponding to a point $(x, y, z)=(r \cos u,(R+$ $r \sin u) \sin v,(R+r \sin u) \cos v)$ in the Euclidean space $\mathbb{R}^{3}$, where $2 R$ is the center-to-center spacing between the discs $D_{1}$ and $D_{2}$, see Fig. 11 in the Appendix A.

Following the original proposal of electromagnetic wormholes by Greenleaf et al. [7], let us now consider the blowup of the centerline of the toroid which goes through the centers of $D_{1}$ and $D_{2}$, onto a toroidal coating using the transform $r^{\prime}=a+r(b-a) / b, u^{\prime}=u$, and $v^{\prime}=v$. Here, $b$ and $a$ are the radii of the circles that form the outer and inner boundaries of the cloaking region, respectively, in toroidal coordinates. Using the transformational plasmonics tools [17], we obtain

$$
\begin{aligned}
\varepsilon_{r r} & =\mu_{r r}=\frac{r-a}{r} \frac{(b-a) R+b(r-a) \sin u}{(b-a)(R+r \sin u)}, \\
\varepsilon_{u u}=\mu_{u u} & =\frac{r}{r-a} \frac{(b-a) R+b(r-a) \sin u}{(b-a)(R+r \sin u)}, \\
\varepsilon_{v v}=\mu_{v v} & =\frac{b^{2}}{b-a} \frac{r-a}{r} \frac{R+r \sin u}{(b-a) R+b(r-a) \sin u} .
\end{aligned}
$$

For a toroidal cloak, the angles $u$ and $v$ vary in the range $(-\pi, \pi)$ and $(0,2 \pi)$. However, for a plasmonic analog of the electromagnetic wormhole like Fig. 1, we only need the upper half part of a toroidal cloak, that is, $u$ varies between 0 and $\pi$. If on the contrary we concentrate on the toroidal cloak lying on the metal surface, see Fig. 2, we now need to tilt the toroidal cloak by an angle of $\pi / 2$ and further cut it in two halves along the $z$ axis, so that it is now $v$ which varies from 0 to $\pi$.

\section{HOMOGENIZATION APPROACH FOR A BROADBAND MULTILAYERED PLASMONIC ANALOG TO THE ELECTROMAGNETIC WORMHOLE}

We would like now to approximate the transformed medium associated with the wormhole by some structured material. We opted for the homogenization approach which should lead to a broadband metamaterial. Indeed, it is fairly easy to extend the design of cylindrical multilayered cloaks originally proposed by Huang et al. [32] to surface plasmon polaritons, see Fig. 5. There is however a further challenge in the present case: We need a multilayered toroidal cloak.

For the construction of the wormhole of type I, we first consider two such cylindrical cloaks located at the holes of the metal plate, see Figs. 6(a) and 6(b). It is reassuring to still observe invisibility in that case. We then consider a structured cylindrical cloak lying on the metal surface, and observe that the scattering of an SPP by a metal obstacle is much reduced, see Figs. 6(c) and 6(d). One question that might arise is whether cloaking still works if we now bend the cylindrical cloaks and whether the wormhole is broadband. We show in Fig. 7 that these results still hold at $800 \mathrm{~nm}$ for a structured toroidal ring which shows that our broadband homogenization approach to this problem is legitimate. Moreover, it is clear from Fig. 7 that there is no need to structure the metal below the 
(a)
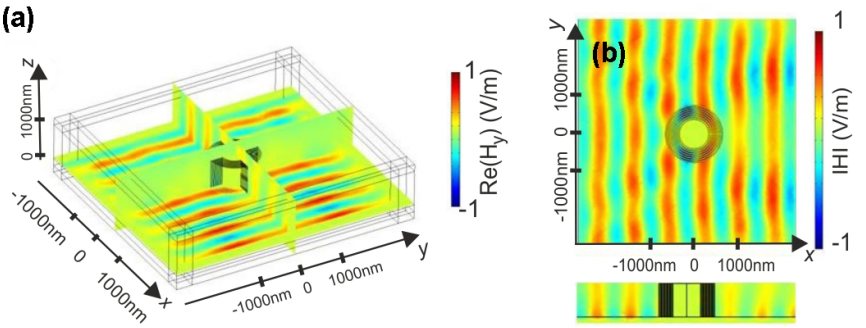

(c)

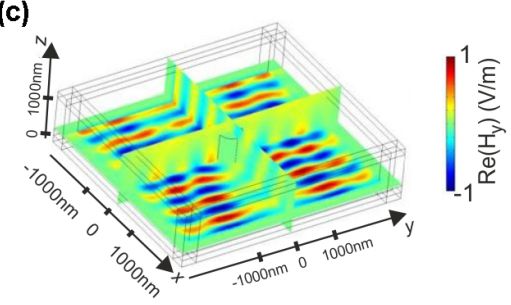

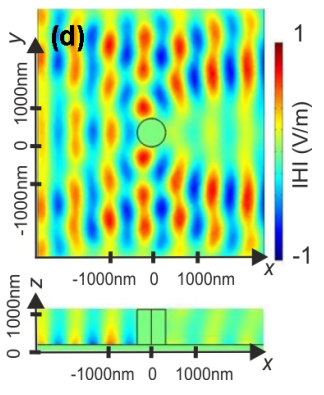

FIG. 5. (Color online) Structured cylindrical magnetic cloak: (a), (b) A SPP incident upon a multilayered cylindrical plasmonic cloak at $700 \mathrm{~nm}$. The material parameters of the layered cloak can be found in [33], wherein a two-dimensional case was considered. (c), (d) The same is plotted for comparison for an obstacle on its own. The reduced backward and forward scattering in (a) and (b) is noted.

air-dielectric interface. This suggests a wormhole of type II might consist only of homogeneous isotropic dielectric layers and might be therefore easier to manufacture than the wormhole of type I. However, the result in Fig. 7 does not clearly show the effect of the curvature of the cloak. In order to give a global overview of cloaking for the dielectric toroidal ring, we show in Fig. 8 that a line source emitting a concentric SPP at the metal surface is not perturbed by the presence of a structured toroidal ring. Importantly, all the previous results were obtained for visible light. We are now ready
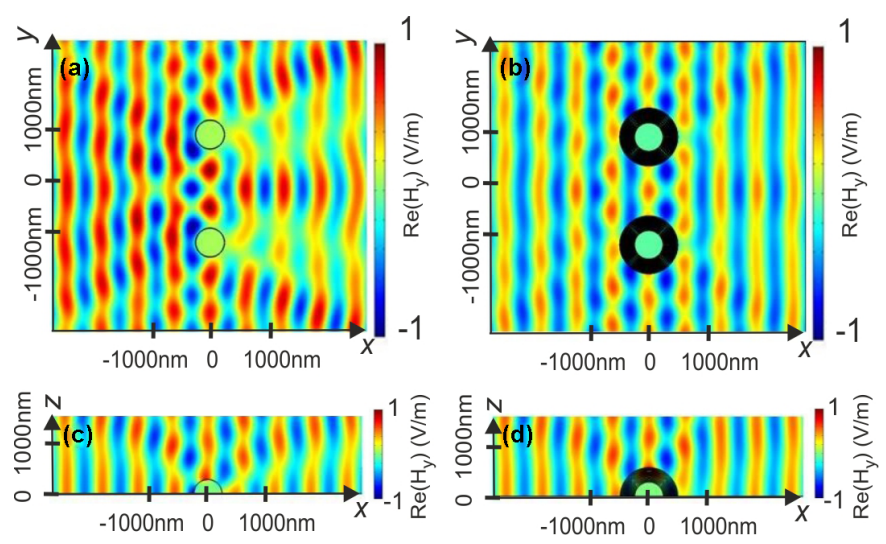

FIG. 6. (Color online) Structured cylindrical magnetic and dielectric cloaks: (a), (b) Top view of the same configuration as in Fig. 5 for two twin cylindrical magnetic cloaks (exemplifying the control of the SPP on the metal surface). (c), (d) Side view of a SPP incident from left upon a cylindrical metal bump on a metal surface on its own (c) and when it is surrounded by a cylindrical dielectric cloak (d) with same parameters as in (b) but lying on the metal surface (exemplifying the control of SPP above the metal surface). The reduced scattering in (b) and (d) is noted.
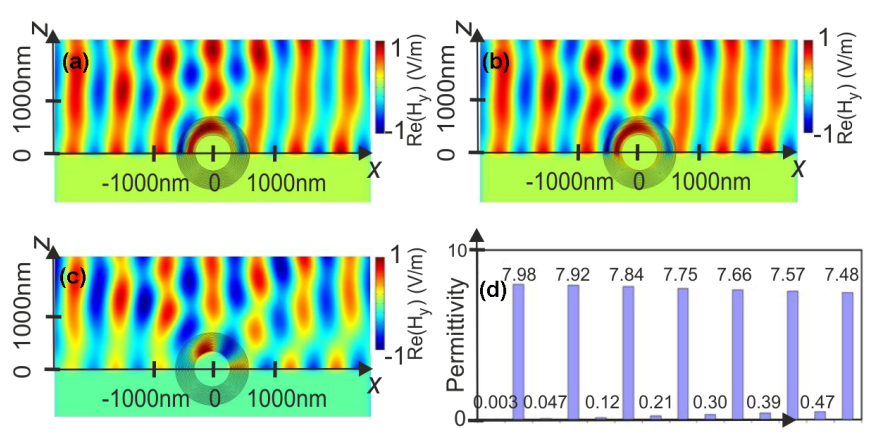

FIG. 7. (Color online) A SPP Gaussian beam with a waste of $2100 \mathrm{~nm}$ launched at $800 \mathrm{~nm}$ is incident upon the structured dielectric wormhole: (a) Side view of a multilayered dielectric wormhole surrounding a metal obstacle. (b) Side view of the same multilayered dielectric wormhole, when we complement it with concentric layers of metals in the metal plate. (c) Side view for the metal obstacle on its own. (d) Values for $\varepsilon$ inside the 14 homogeneous layers. The similar scattering in (a) and (b) is noted.

to theoretically investigate the design of structured toroidal cloaks via effective medium (homogenization) theory.

\section{A. Reduced parameters for a toroidal cloak}

In order to simplify the design of the wormhole, we proceed in a way similar to what was done to obtain a reduced set of material parameters for cylindrical cloaks in [34]. Using the transformational plasmonics tools, we obtain

$$
\begin{aligned}
\varepsilon_{r r} & =\mu_{r r}=\frac{r-a}{r} f(r, u), \quad \varepsilon_{u u}=\mu_{u u}=\frac{r}{r-a} f(r, u), \\
\varepsilon_{v v} & =\mu_{v v}=\frac{b^{2}}{(b-a)^{2}} \frac{r-a}{r f(r, u)},
\end{aligned}
$$

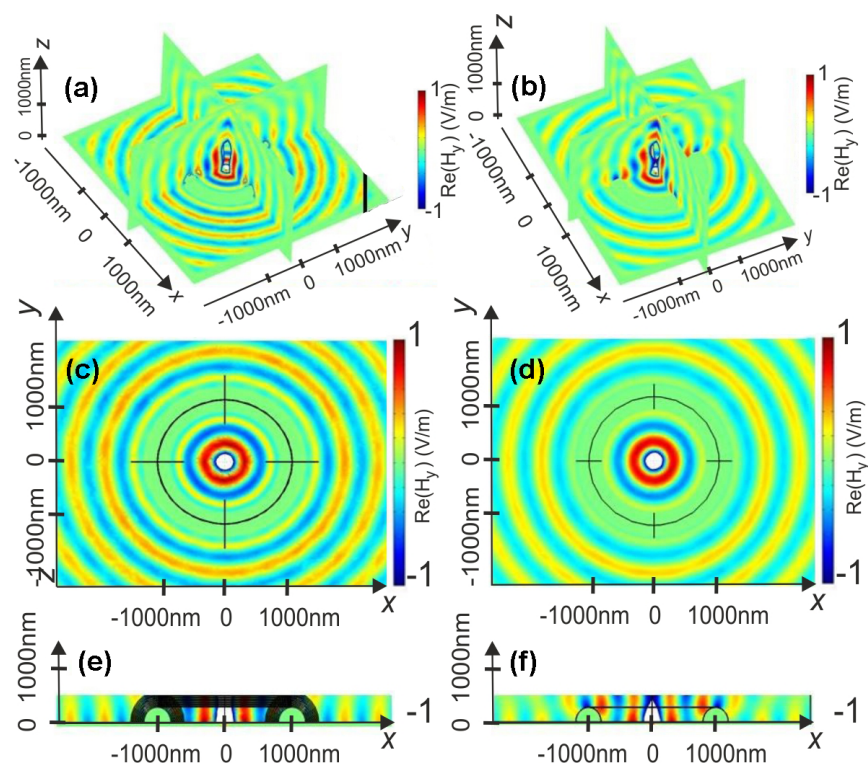

FIG. 8. (Color online) A line source generating a concentric SPP placed in the center of the system at a wavelength of $700 \mathrm{~nm}$ is much less perturbed by the presence of the wormhole (as shown by the isotropic wave pattern in the view from above and the side view for the evanescent part of the SPP) than by the presence of an obstacle on its own. 
where $f(r, u)=\frac{(b-a) R+b(r-a) \sin u}{(b-a)(R+r \sin u)}$, which leads us to the set of reduced parameters

$$
\begin{aligned}
& \varepsilon_{r r}=\mu_{r r}=\left(\frac{r-a}{r}\right)^{2}, \quad \varepsilon_{u u}=\mu_{u u}=1, \\
& \varepsilon_{v v}=\mu_{v v}=\frac{b^{2}}{(b-a)^{2}}
\end{aligned}
$$

that preserve the wave trajectories, but induce a slight impedance mismatch on the wormhole outer boundary.

For a toroidal cloak, both angles $u$ and $v$ vary in the range $(0,2 \pi)$. However, for a plasmonic analog of the electromagnetic wormhole, see Fig. 1(a), we only need the upper half of a toroidal cloak, that is, $u$ varies between 0 and $\pi$. If on the contrary we concentrate on the toroidal cloak lying on the metal surface, see Fig. 2, we now need to tilt the toroidal cloak by an angle of $\pi / 2$ and further cut it in two halves along the $z$ axis, so that it is now $v$ which varies from 0 to $\pi$.

\section{B. Homogenized parameters for a toroidal cloak}

We note that if the toroidal cloak component of the wormhole consists of an alternation of two homogeneous isotropic layers of thicknesses $d_{A}$ and $d_{B}$ and permittivity $\varepsilon_{A}=\lambda_{A}^{-1}, \varepsilon_{B}=\lambda_{B}^{-1}$ and permeability $\mu_{A}=\lambda_{A}$ and $\mu_{B}=\lambda_{B}$, we have

$$
\frac{1}{\lambda_{r r}}=\frac{1}{1+\eta}\left(\frac{1}{\lambda_{A}}+\frac{\eta}{\lambda_{B}}\right), \quad \lambda_{u u}=\lambda_{v v}=\frac{\lambda_{A}+\eta \lambda_{B}}{1+\eta},
$$

where $\eta=d_{B} / d_{A}$ is the ratio of thicknesses for layers $A$ and $B$ and $d_{A}+d_{B}=1$.

We shall use the previous homogenized formulas in order to approximate the reduced parameters (4).

We report in Fig. 9 some computations for a SPP incident at $700 \mathrm{~nm}$ upon the structured magnetic wormhole consisting

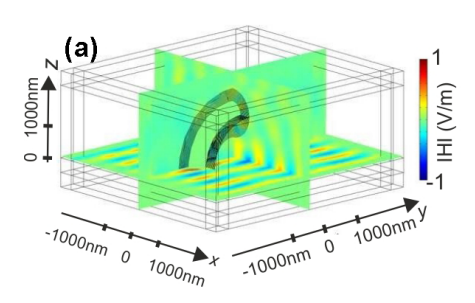

(c)
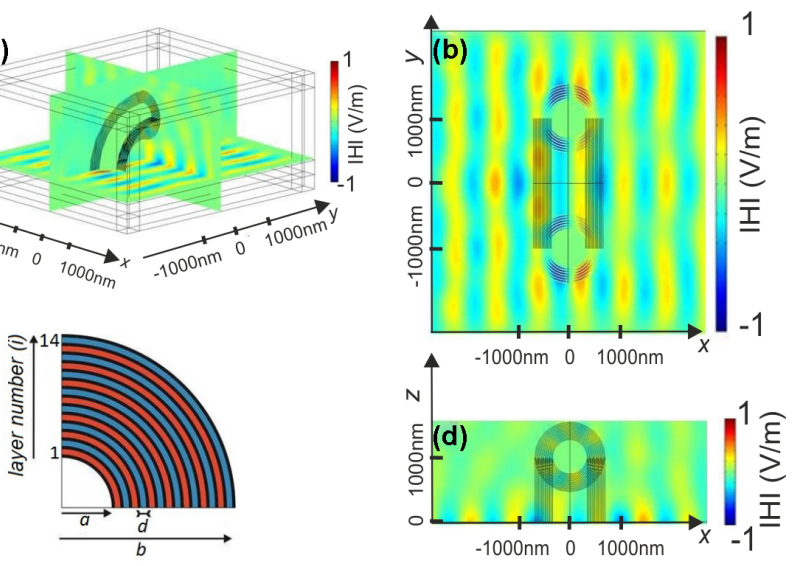

FIG. 9. (Color online) Structured magnetic wormhole: A SPP Gaussian beam with a waste of $2100 \mathrm{~nm}$ incident upon a multilayered cylindrical wormhole at $700 \mathrm{~nm}$ smoothly bent around a metal toroidal obstacle. The permeability in every layer (of identical thickness 60 $\mathrm{nm})$ is given by $\mu_{i}=[0,0032 ; 7,97 ; 0,0467 ; 7,91 ; 0,121 ; 7,84 ; 0,207$; $7,75 ; 0,297 ; 7,66 ; 0,386 ; 7,57 ; 0,473 ; 7,48]$ (from the inner to the outer layer). (a) Three-dimensional plot of the real part of the magnetic field. (b) Top view. (c) Diagrammatic view of the device. (d) Side view. The color scale has been normalized. (a)

(b)
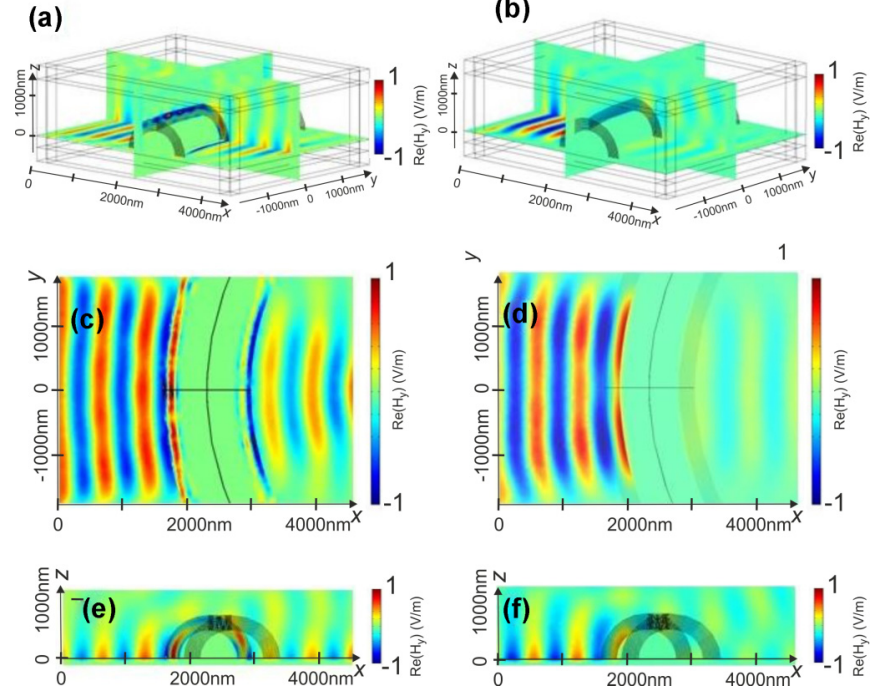

FIG. 10. (Color online) Structured dielectric wormhole: SPP Gaussian beam with a waste of $2100 \mathrm{~nm}$ incident at $700 \mathrm{~nm}$ upon a metal toroidal obstacle dressed with the wormhole (left panel) and on its own (right panel). The permittivity in every layer (of identical thickness $60 \mathrm{~nm}$ ) is given by $\varepsilon_{i}=[0,0032 ; 7,97 ; 0,0467 ; 7,91 ; 0,121$; $7,84 ; 0,207 ; 7,75 ; 0,297 ; 7,66 ; 0,386 ; 7,57 ; 0,473 ; 7,48]$ (from the inner to the outer layer). (a), (b) Three-dimensional plot. (c), (d) View from above. (e), (f) Side view. The color scale has been normalized.

of an alternation of homogeneous magnetic layers specified in the figure caption, which could be achieved as in [33]. If we now tilt the wormhole by an angle $\pi / 2$, a similar design holds with an alternation of dielectric homogeneous layers, see Fig. 10. The performance of this dielectric SPP analog to an electromagnetic wormhole is further ascertained by placing a plasmonic source in its center and observing the unperturbed concentric wavefronts emanating from the source, see Fig. 8.

\section{CONCLUSION}

In conclusion, we have studied analytically and numerically the extension of wormholes to the domain of surface plasmon waves propagating at the interface between metal and dielectric or air. These waves obey the Maxwell equations at a flat interface and are evanescent in the transverse direction, so that, the problem we have treated is somewhat a two-dimensional plasmonic analog of the electromagnetic wormhole designed by Greenleaf et al. [7]: It is enough to consider the wormhole as a manifold in a three dimensional Euclidean space for applications in plasmonics. Nevertheless, our numerical computations based on the finite element method take into account the three dimensional features of the problem, such as the plasmon polarization and the jump of permittivity at the interface between metal and metamaterial or air which are described by permittivities of opposite sign.

Electromagnetic wormholes [7] represent a fascinating paradigm, but were initially thought of as an abstract metamaterial bridging two spherical holes, thereby requiring a further dimension for the invisible tunnel, and moreover no permittivity and permeability tensors were derived from a specific structured design. We have transposed this idea to 
the area of surface plasmon polaritons, with two illustrative examples of plasmonic analogs of electromagnetic wormholes (SPP analogs of volume electromagnetic effects described in [7]): An invisible handlebody and an invisible ring over metal surfaces. We further proposed multilayered versions of these metamaterials which work over a finite range of visible frequencies, hoping to foster experiments in the emerging field of transformational plasmonics. Potential applications might be in safer communications and intraship technologies.

\section{ACKNOWLEDGMENTS}

S.G. is thankful for a European funding through ERC Starting Grant ANAMORPHISM.

\section{APPENDIX A: SOFT-HARD CONDITIONS}

In what follows, we numerically demonstrate that enforcing some nonnatural conditions on the inner boundary, known as soft-hard (SH) conditions:

$$
\mathbf{e}_{\theta} \cdot \mathbf{E}=0, \quad \mathbf{e}_{\theta} \cdot \mathbf{H}=0,
$$

where $\mathbf{e}_{\theta}$ is the angular unit vector and $E$ and $H$ the local electric and magnetic fields, respectively, leads to a farfield pattern very similar to perfect magnetic conditions (Figs. 11, 12, and 13).

For this, we extend the $\mathrm{SH}$ conditions to our wormhole as per

$$
\mathbf{e}_{w} \cdot \mathbf{E}=0, \quad \mathbf{e}_{w} \cdot \mathbf{H}=0,
$$

where $\mathbf{e}_{w}$ is the angular unit vector in the toroidal basis $(r, w, v)$.

The main difficulty is to impose such condition in a finite element algorithm, in our case, Comsol Multiphysics. We start with the case of an $x$-axis torus on a metal plate. The

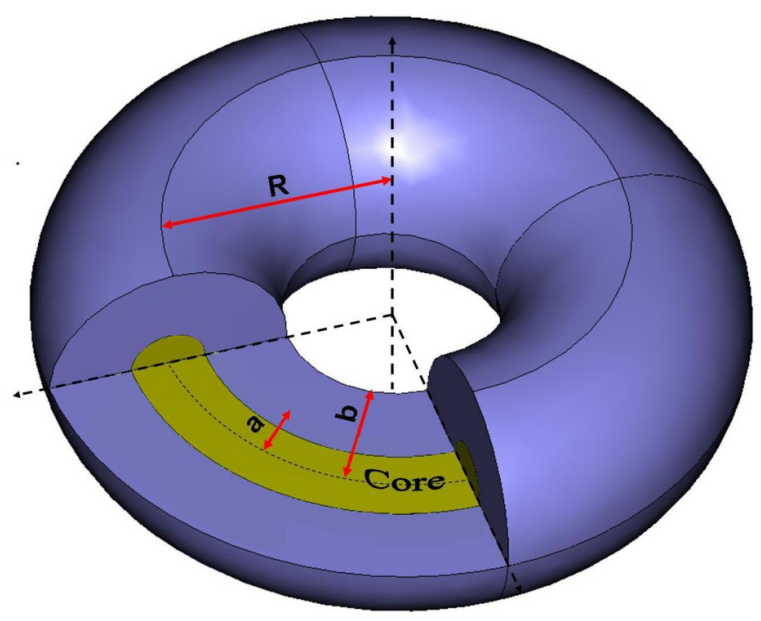

FIG. 11. (Color online) Diagrammatic view of a toroidal wormhole: $R$ is the major radius of the torus, and $a$ and $b$ are, respectively, the radii of the inner and outer boundaries of the toroidal wormhole. The core (an isotropic dielectric medium shown in yellow color) corresponds to the invisible waveguide and the coating (the transformed medium described by tensors of permittivity and permeability shown in blue color) allows the control of the surrounding electromagnetic field, e.g., SPPs. parametrization is the following one:

$$
\begin{aligned}
& x=r \cos (w), \\
& y=(R+r \sin (w)) \sin (v), \\
& z=(R+r \sin (w)) \cos (v) .
\end{aligned}
$$

The vector $\mathbf{e}_{w}$ is given by

$$
\begin{aligned}
& x=-r \sin (w), \\
& y=r \cos (w) \sin (v), \\
& z=r \cos (w) \cos (v) .
\end{aligned}
$$

\section{APPENDIX B: FINITE ELEMENT MODEL AND ILLUSTRATIVE NUMERICAL EXAMPLES}

We opted for Nedelec or edge finite elements, which naturally fulfill the tangential continuity of the electromagnetic field across interfaces. Put in a more mathematical way, mixed finite elements are part of a discrete algebraic-geometricdifferential structure of finite element shape functions invented by Whitney [35] which assign degrees of freedom to simplices of a given mesh: nodes, edges, facets, tetrahedra. This structure, the so-called Whitney complex, closely matches a continuous structure made of four-vector subspaces of L2 and of three differential operators grad, curl, and div, which is known as the de Rham complex. This complex is called an exact sequence if the image of each operator domain of this structure is exactly the kernel of the next operator. Clearly, this statement depends upon the topological properties of the domains, such as connectivity assumptions. This is why we choose the point of view of differential geometry in this article: It makes the numerical modeling much easier to handle. In this way, we indeed consider a simplicial mesh on a three-dimensional manifold $W$, that is, a set of tetrahedra which 2 by 2 have in common either a full facet, or a full edge, or a node (vertex), or nothing, and whose set union is $W$.

In Comsol Multiphysics, we decided to solve the problem for the magnetic component $\mathbf{H}$ of the electromagnetic field:

$$
\nabla \times\left(\underline{\underline{\varepsilon}}^{-1} \nabla \times \mathbf{H}\right)=\omega^{2} \varepsilon_{0} \mu_{0} \underline{\underline{\mu}}=
$$

where $\mu_{0} \varepsilon_{0}=c^{-2}$, with $c$ the speed of light in a vacuum and $\underline{\varepsilon}$ and $\mu$ are tensors of relative permittivity and permeability proportional to the identity matrix outside the wormhole and fully anisotropic and highly heterogeneous within the wormhole. Here, the unknown $\mathbf{H}=\mathbf{H}_{i}+\mathbf{H}_{d}$, where $\mathbf{H}_{i}$ is the incident field and $\mathbf{H}_{d}$ is the diffracted field which satisfies the usual outgoing wave conditions (to ensure existence and uniqueness of the solution). The weak formulation associated with (B1) is discretized using second order finite edge elements (or Whitney forms) which behave nicely under geometric transforms (pull-back properties) [36].

Since the above equation is taken in a weak sense, it contains within it the transmission conditions across interfaces between different media. In particular, the tangential continuity of the solution across the inner boundary of the wormhole reads as

$$
\mathbf{n} \cdot\left(\underline{\underline{\varepsilon}}^{-1} \nabla \times \mathbf{H}\right)=0,
$$

where $\mathbf{n}$ is the unit outward normal to the boundary. 

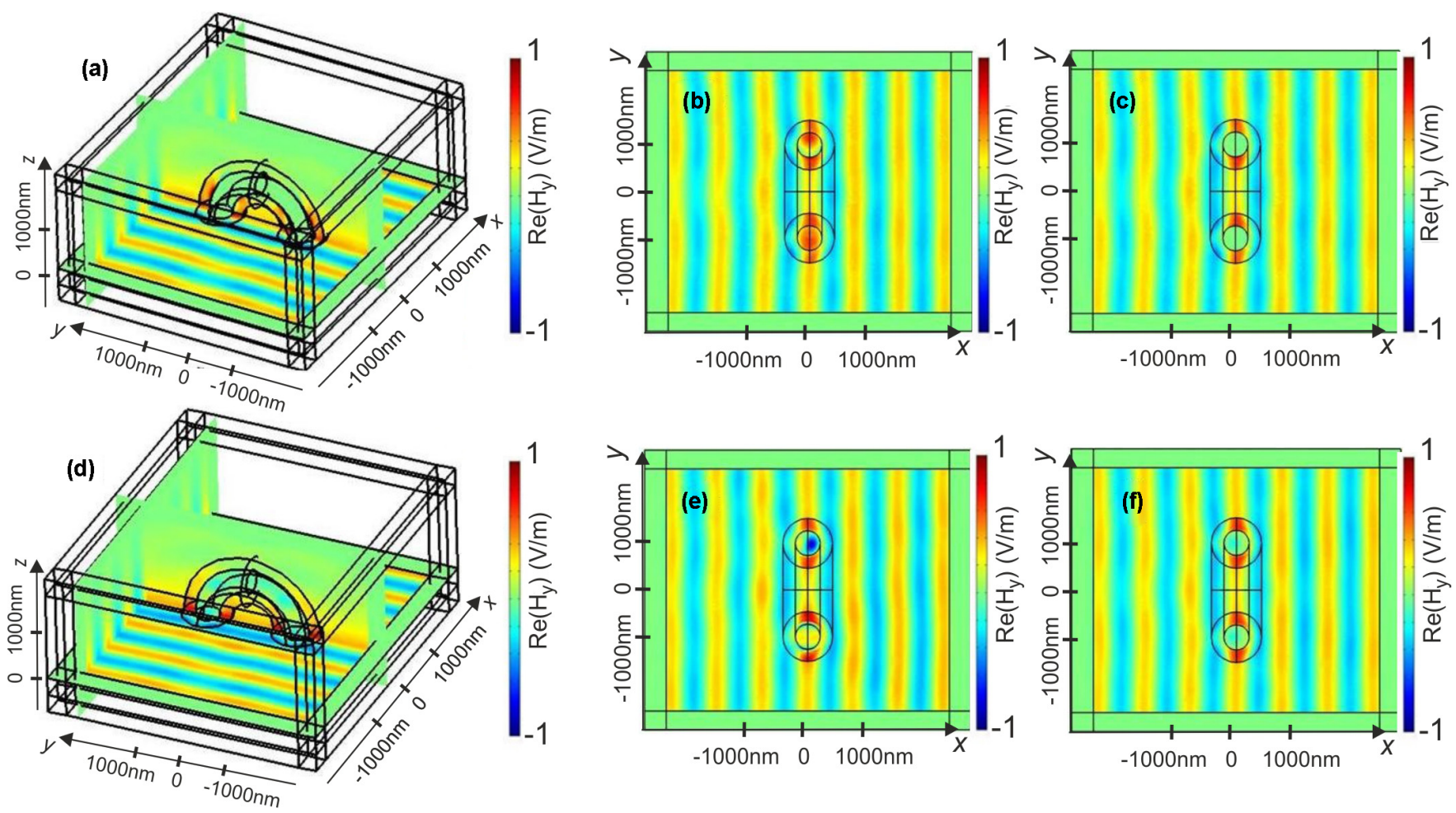

FIG. 12. (Color online) Perfect magnetic conductor (PMC) versus SH lining condition at $700 \mathrm{~nm}$ [The incident field is set as described in Eq. (1) of the accompanying paper with the field amplitude $H_{y_{i}}=1$.]: (a) 3D plot of the total magnetic field for the PMC condition on the inner boundary of the wormhole without a propagating field inside the core. (b) Two-dimensional (2D) plot of the total magnetic field for the PMC condition on the inner boundary of the wormhole with a propagating field inside the core. (c) 2D plot of the total magnetic field for the PMC condition on the inner boundary of the wormhole without a propagating field inside the core. (d) 3D plot of the total magnetic field for the SH lining condition on the inner boundary of the wormhole without a propagating field inside the core. (e) $2 \mathrm{D}$ plot of the total magnetic field for the SH lining condition on the inner boundary of the wormhole with a propagating field inside the core. (f) $2 \mathrm{D}$ plot of the total magnetic field for the SH lining condition on the inner boundary of the wormhole without a propagating field inside the core.

The SH condition (A2) should therefore be reexpressed into

$$
\mathbf{e}_{w} \cdot\left(\underline{\varepsilon}^{-1} \nabla \times \mathbf{H}\right)=0, \quad \mathbf{e}_{w} \cdot \mathbf{H}=0 .
$$

Such conditions can be enforced in COMSOL using the built-in constraint dialog box in the boundary settings-equation system, see [37]. However, other finite element packages may also be able to handle this model.

The permittivity and permeability in the wormhole are easily deduced through the relationship $\underline{\underline{\varepsilon}}=\underline{\underline{\mu}}=\mathbf{T}$ [36], where the entries of the transformation matrix $\mathbf{T}$ are given in the next

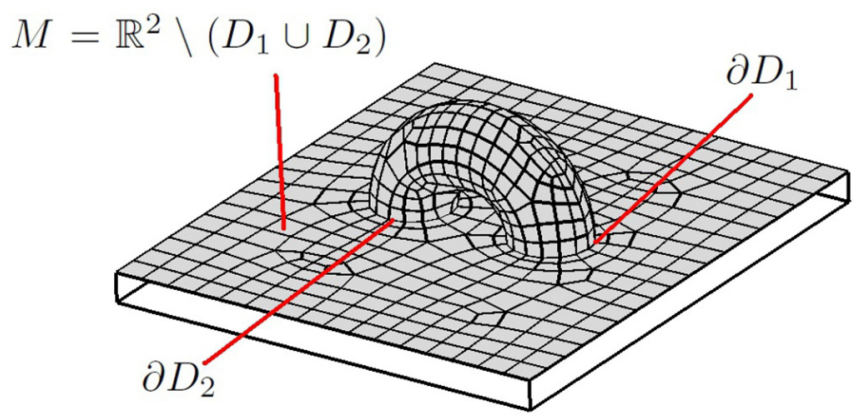

FIG. 13. (Color online) Three-dimensional tetrahedral mesh for the two-dimensional manifold $W=M \cup T$, where $M=\mathbb{R}^{2} \backslash\left(D_{1} \cup\right.$ $D_{2}$ ) for the plasmonic analog of electromagnetic wormhole 1 , where $D_{1}$ and $D_{2}$ are the remote holes (discs) in the metal plate. section. The implementation of the SH lining condition on the inner boundary follows from the conditions (B3).

Let us now describe the numerical results obtained using the three types of boundary conditions on the inner boundary of the wormhole (PMC, SH lining, and transmission conditions). In Fig. 9, we implemented the PMC condition (upper panels) and the SH lining condition (lower panels) and further applied an electromagnetic field in the inner region). It can be seen that there are no significant differences between the upper and lower panels, that is, for the $y$-polarized magnetic field. From this, we can conclude that PMC conditions work well enough regarding the scattered magnetic field. However, if we now focus our attention on the (one nonvanishing) $z$ component of the electric field, cf. Fig. 14, it transpires that SH lining conditions and PMC conditions lead to a dramatically different behavior of the field inside the core of the wormhole: in the $\mathrm{SH}$ case, the magnitude of the field is large inside the core, while for PMC (and transmission) conditions it vanishes (as one would expect for an invisibility cloak). Importantly, the electric field is nonsingular across the interface between the coating and the core for both PMC and SH conditions, while the transmission condition leads to a singular field (indeed, the $\mathbf{T}$ matrix is singular at this interface. It is also illuminating to plot all the fields components. In Fig. 15, one can see that the $H_{y}$ component is indeed the dominant one, which is not surprising as we launched a $y$-polarized SPP. However, the other two 

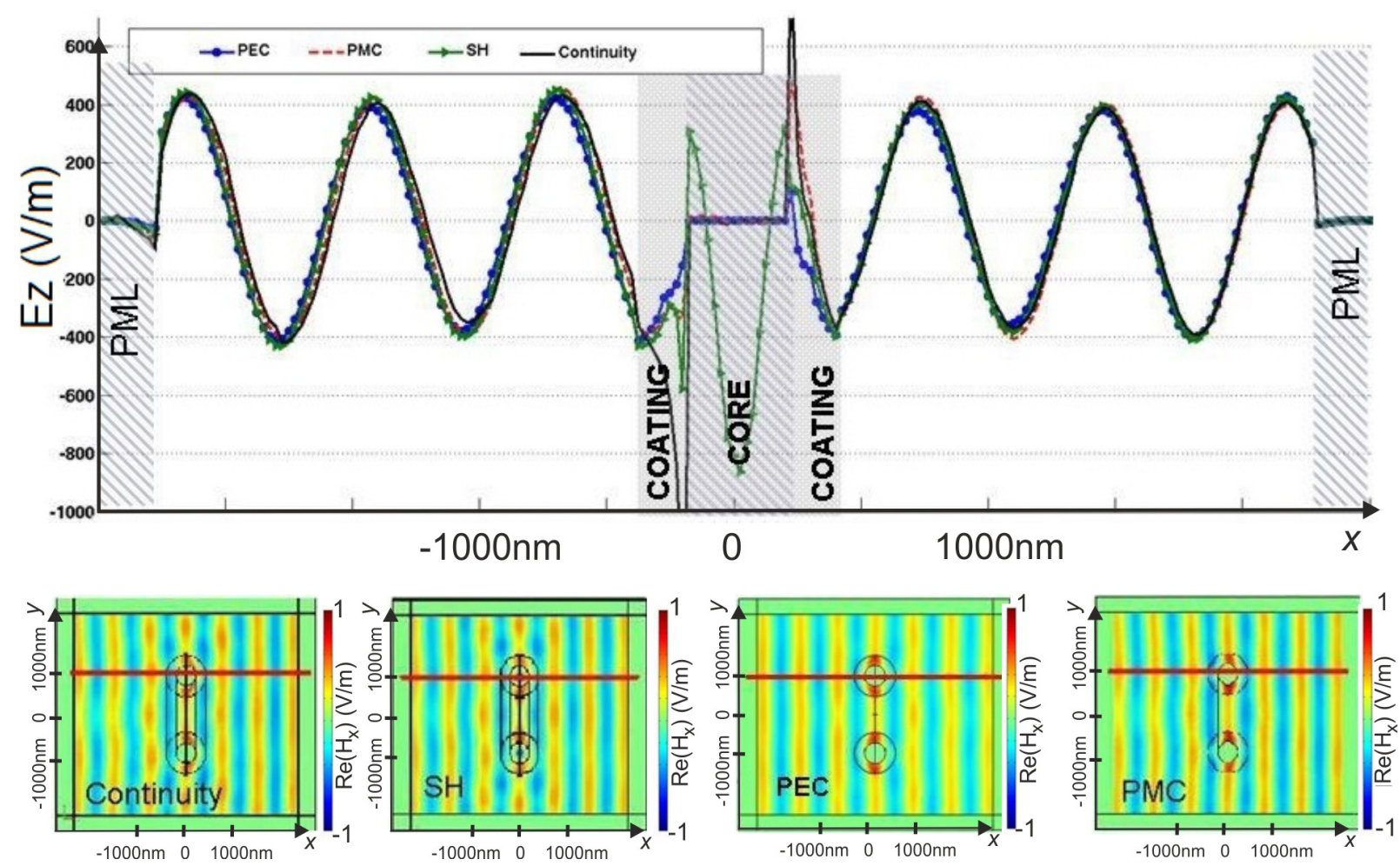

FIG. 14. (Color online) Profile of $E_{z}$ in the plane $z=0$ along the red line shown in the inset, for three conditions on the inner boundary of the toroidal wormhole: soft-hard lining condition (dotted green curve), continuity condition (continuous black curve), perfect electric conductor (PEC in blue), and perfect magnetic conductor (PMC in dashed red curve). We note for the PMC condition, all that along this line both components $E_{x}$ and $E_{y}$ vanish for the $\mathrm{SH}$ condition. The $E_{z}$ component is not set to 0 for $\mathrm{SH}$ condition.

components of the magnetic field do not vanish within the wormhole: indeed they couple inside the spatially varying ansitropic coating: one should then note that the $z$ component of the electric field is stronger than the other two components, as it is essentially coming from the $x$ and $y$ components of the magnetic field (through rotation by the curl operator). The fact that the $E_{z}$ field is fairly large within the core of the wormhole is reminiscent of the almost trapped states in the work of [38].

From the mathematical viewpoint, the idea of soft-hard boundary conditions is very appealing [39] as it regularizes the electromagnetic field (thereby ensuring a well-posed problem in usual energy normed functional spaces). However, from
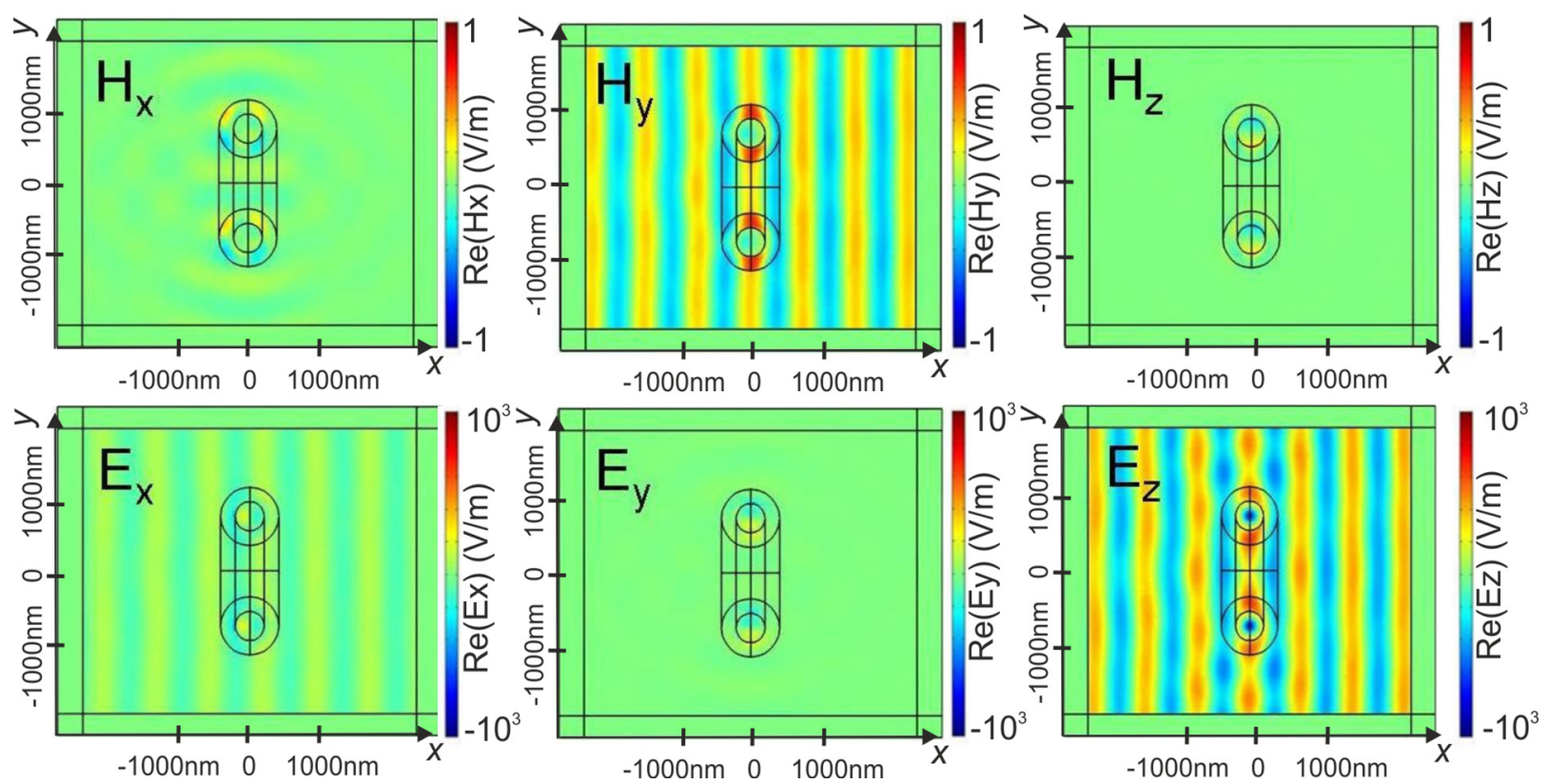

FIG. 15. (Color online) All components of the electromagnetic field for the SH condition. Note the factor of 1000 between the color scales for the magnetic and electric field components. 

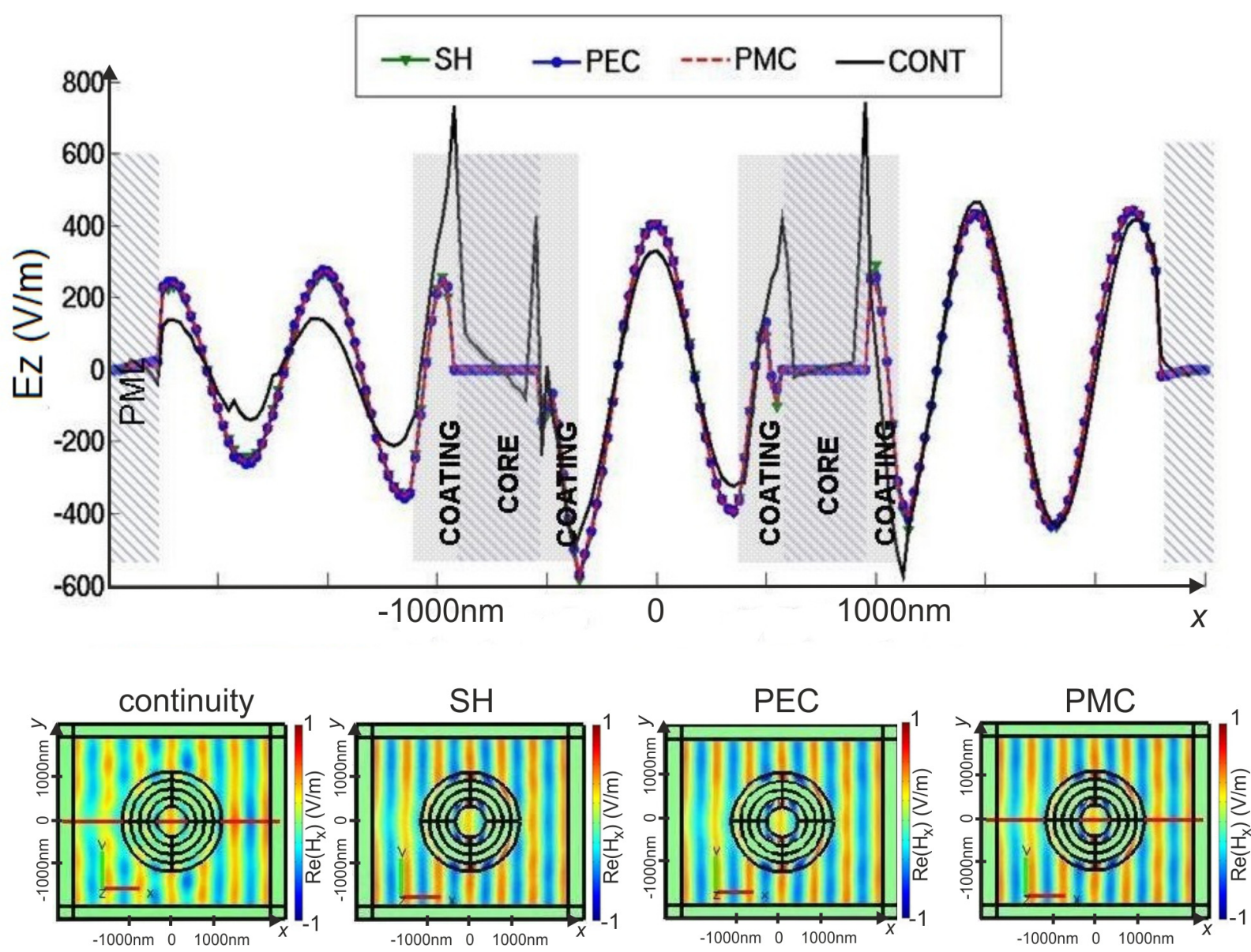

FIG. 16. (Color online) Profile of $E_{z}$ in the plane $z=0$ along the red line shown in the inset, for three conditions on the inner boundary of the toroidal wormhole: soft-hard lining condition (green curve with triangles), continuity condition (continuous black curve), perfect electric conductor (PEC in dotted blue curve), and perfect magnetic conductor (PMC in dashed red curve).

the more practical viewpoint, the PMC condition has the advantage of preventing any field to penetrate the core region. Indeed, when an electromagnetic mode propagates within the core of the wormhole, it would inevitably interfere with the exterior field which is nonvanishing in case of SH lining conditions. Hence, the whole aspect of an invisible tunnel would be spoiled.

\section{APPENDIX C: MAGNETIC TORUS}

A few words would be in order regarding the feasibility of the magnetic torus. Wood and Pendry have proposed in 2007 a route towards magnetic metamaterials operating at low frequencies with a structure based on superconducting elements [40]. This theoretical proposal has been since then experimentally validated [41] with a magnetic cloak. The range of parameters required for $\mu$ in Fig. 6 is as for $\varepsilon$ in Fig. 7, and is therefore within experimental reach. We show in Figs. 16 and 17 the counterpart of Figs. 14 and 15 for the dielectric wormhole.

\section{APPENDIX D: DEVELOPED EXPRESSION FOR \\ THE TRANSFORMATION MATRIX IN CARTESIAN COORDINATES}

The toroidal coordinates $(r, v, w)$ can be expressed in terms of Cartesian coordinates $(x, y, z)$ as follows:

$$
\begin{gathered}
r=\frac{\sqrt{\rho\left(y^{2} \rho-2 y^{2} R+z^{2} \rho-2 z^{2} R+R^{2} \rho+x^{2} \rho\right)}}{\rho}, \quad \text { with } \rho=\sqrt{y^{2}+z^{2}}, \\
v=\arctan \left(\frac{y}{z}\right), w=\arctan \left(\frac{(\rho-R) \rho\left[\rho\left(y^{2} \sqrt{y^{2}+z^{2}}-2 y^{2} R+z^{2} \rho-2 z^{2} R+R^{2} \rho+x^{2} \rho\right)\right]^{-1 / 2}}{x \rho \sqrt{\rho\left(y^{2} \rho-2 y^{2} R+z^{2} \rho-2 z^{2} R+R^{2} \rho+x^{2} \rho\right)}}\right) .
\end{gathered}
$$

A systematic way to identify the tensors of permittivity and permeability associated with the toroidal geometry is actually to consider the Jacobian matrix $\mathbf{J}_{\mathrm{xr}}$ associated with the opposite change of coordinate system. We emphasize the fact that it is 

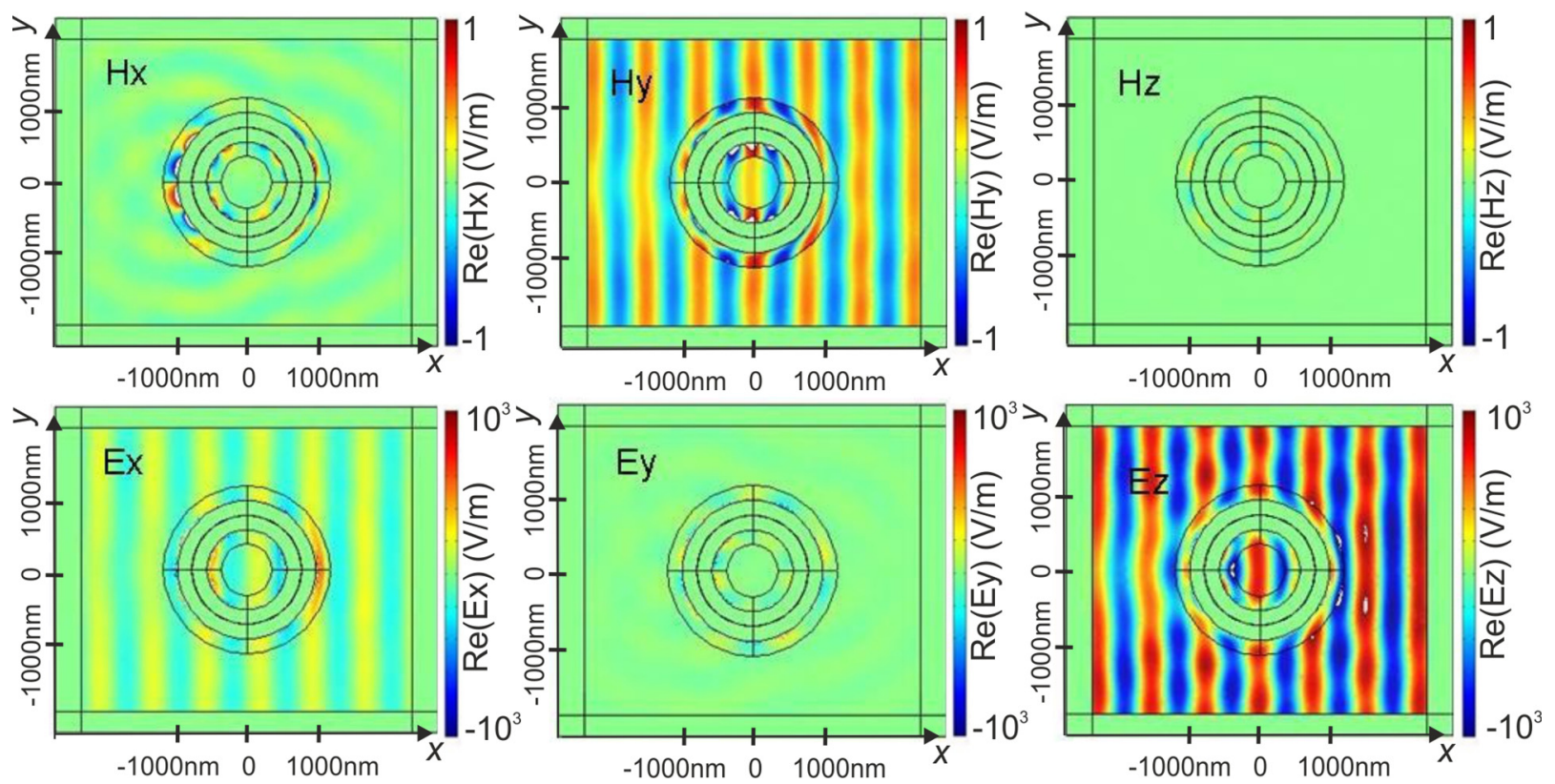

FIG. 17. (Color online) All components of the electromagnetic field for the SH condition. Note the factor of 1000 between the color scales for the magnetic and electric field components.

the transformed domain and coordinate system $(r, v, w)$ that are mapped onto the initial domain with Cartesian coordinates $(x, y, z)$, and not the opposite:

$$
\begin{aligned}
& d x=\frac{\partial x}{\partial r} d r+\frac{\partial x}{\partial v} d v+\frac{\partial x}{\partial w} d w \\
& d y=\frac{\partial y}{\partial r} d r+\frac{\partial y}{\partial v} d v+\frac{\partial y}{\partial w} d w \Longleftrightarrow\left(\begin{array}{c}
d x \\
d y \\
d z
\end{array}\right)=\mathbf{J}_{\mathrm{xr}}\left(\begin{array}{c}
d r \\
d v \\
d w
\end{array}\right) \\
& d z=\frac{\partial z}{\partial r} d r+\frac{\partial z}{\partial v} d v+\frac{\partial z}{\partial w} d w
\end{aligned}
$$

It follows that the transformation rule for expressing the tensors $\varepsilon^{\prime}$ and $\mu^{\prime}$ in the transformed coordinates in terms of the tensors $\underline{\underline{\varepsilon}}$ and $\underline{\underline{\mu}}$ in the Cartesian coordinates is [36]

$$
\begin{aligned}
& \underline{\underline{\varepsilon^{\prime}}}(r, v, w)=\mathbf{J}_{\mathrm{xr}}^{-1} \underline{\underline{\varepsilon}}(x, y, z) \mathbf{J}_{\mathrm{xr}}^{-\mathrm{T}}\left|\operatorname{det}\left(\mathbf{J}_{\mathrm{xr}}\right)\right| \\
& \underline{\underline{\underline{\mu}}}(r, v, w)=\mathbf{J}_{\mathrm{xr}}^{-1} \stackrel{\mu}{=}(x, y, z) \mathbf{J}_{\mathrm{xr}}^{-\mathrm{T}}\left|\operatorname{det}\left(\mathbf{J}_{\mathrm{xr}}\right)\right|
\end{aligned}
$$

where $\operatorname{det}\left(\mathbf{J}_{\mathrm{xr}}\right)$ is the determinant of the Jacobian matrix and $\mathbf{J}_{\mathrm{xr}}^{-\mathrm{T}}$ denotes the inverse transpose matrix of $\mathbf{J}_{\mathrm{xr}}$.

When the original permittivity and permeability matrices are proportional to the identity matrix, which is our case, their transformed counterparts are given by

$$
\underline{\varepsilon^{\prime}}=\varepsilon \mathbf{T}_{\mathrm{xr}}^{-1}, \quad \underline{\underline{\mu^{\prime}}}=\mu \mathbf{T}_{\mathrm{xr}}^{-1}, \quad \text { where } \quad \mathbf{T}_{\mathrm{xr}}=\frac{\mathbf{J}_{\mathrm{xr}}^{T} \mathbf{J}_{\mathrm{xr}}}{\left|\operatorname{det}\left(\mathbf{J}_{\mathrm{xr}}\right)\right|} .
$$

Thus far, we have only deduced the transformation matrix for transformed medium associated with toroidal coordinates, but we did not take into account the stretch of toroidal coordinates $r^{\prime}=a+r(b-a) / b$ used to blowup the centerline of the toroid in order to design the invisible handlebody of the wormhole. It thus remains to compute the transformation matrix $\mathbf{T}$ as expressed in the Cartesian coordinates $\left(x^{\prime}, y^{\prime}, z^{\prime}\right)$ associated with the cylindrical cloak, we start with the product of three elementary Jacobians :

$$
\mathbf{J}_{x x^{\prime}}=\mathbf{J}_{\mathrm{xr}} \mathbf{J}_{r r^{\prime}} \mathbf{J}_{r^{\prime} x^{\prime}}
$$

where $\mathbf{J}_{\mathrm{xr}}$ (resp. $\mathbf{J}_{r^{\prime} x^{\prime}}$ ) is the Jacobian associated with the change to toroidal coordinates $[r(x, y, z), v(x, y, z), w(x, y, z)]$ (resp. transformed Cartesian coordinates $\left[x^{\prime}\left(r^{\prime}, v^{\prime}, w^{\prime}\right)\right.$, $\left.\left.y^{\prime}\left(r^{\prime}, v^{\prime}, w^{\prime}\right), z^{\prime}\left(r^{\prime}, v^{\prime}, w^{\prime}\right)\right]\right)$ and where

$$
\mathbf{J}_{r r^{\prime}}=\operatorname{diag}\left(\frac{b}{b-a}, 1,1\right)
$$

are the radially contracted toroidal coordinates, which have the same form as the one proposed in [1] for stretched polar coordinates in the context of cylindrical cloaks. Importantly, this transform was used by You et al. [42] for the design of toroidal cloaks in the context of photonics, which leads to other kinds of metamaterials.

Finally, the material properties of the toroidal invisible handlebody that bridges the holes $D_{1}$ and $D_{2}$ on the metal surface are described by the transformation matrix

$$
\begin{aligned}
& \stackrel{\varepsilon^{\prime \prime}}{=}=\varepsilon \mathbf{T}^{-1}, \quad \underline{\underline{\mu^{\prime \prime}}}=\mu \mathbf{T}^{-1}, \\
& \text { where } \quad \mathbf{T}:=\mathbf{T}_{x x^{\prime}}=\frac{\mathbf{J}_{x x^{\prime}}^{T} \mathbf{J}_{x x^{\prime}}}{\left|\operatorname{det}\left(\mathbf{J}_{x x^{\prime}}\right)\right|} .
\end{aligned}
$$

Overall, the entries of the symmetric transformation matrix $\mathbf{T}$ are as follows:

$$
\begin{aligned}
T_{11} & =-\left[-\beta^{2} \cos (w)^{2}+2 r \cos (w)^{2} \beta-r^{2}\right][R \alpha+r \sin (w)-\sin (w) \beta] /\{r(r-\beta) \alpha[R+r \sin (w)]\} \\
T_{12}= & \beta(-\beta+2 r)\left[-r \sin (w) R+R \sin (w) \beta+R \sin (w) r \alpha-R^{2} \alpha+r^{2}-r \beta-r^{2} \cos (w)^{2}+r \cos (w)^{2} \beta\right] \\
& \times[\sin (v) \sin (w) \cos (w)] /\left[r(r-\beta) \alpha\left(r^{2} \cos (w)^{2}-r^{2}+R^{2}\right)\right],
\end{aligned}
$$




$$
\begin{aligned}
T_{13}= & \beta(-\beta+2 r)\left[-r \sin (w) R+R \sin (w) \beta+R \sin (w) r \alpha-R^{2} \alpha+r^{2}-r \beta-r^{2} \cos (w)^{2}+r \cos (w)^{2} \beta\right] \\
& \times[\cos (v) \sin (w) \cos (w)] /\left[r(r-\beta) \alpha\left(r^{2} \cos (w)^{2}-r^{2}+R^{2}\right)\right],
\end{aligned}
$$

$$
\begin{aligned}
T_{22}= & -\left[\left(2 \cos (v)^{2} \cos (w)^{2} R \sin (w) \alpha \beta^{3}+2 \cos (w)^{2} \cos (v)^{2} R^{2} \beta \alpha^{2} r-6 R \sin (w) \cos (v)^{2} \alpha r^{2} \beta+6 R \sin (w) \cos (v)^{2} \alpha r \beta^{2}\right.\right. \\
& -2 \alpha^{2} R^{2} r \beta \cos (w)^{2}+6 \cos (w)^{2} \beta^{2} R \sin (w) r \alpha+2 r^{3} \cos (w)^{4} \beta+2 R \sin (w) \alpha \beta^{3}-\beta^{4}+\cos (v)^{2} \beta^{4}+r^{4} \cos (w)^{2} \\
& +4 \beta^{3} r-\alpha^{2} R^{2} \beta^{2}+2 R^{2} \beta \alpha^{2} r-8 \cos (w)^{2} \beta^{3} r-r^{2} \alpha^{2} R^{2}-6 \cos (v)^{2} \cos (w)^{2} R \sin (w) \alpha \beta^{2} r-2 r^{3} \cos (w)^{4} \cos (v)^{2} \beta \\
& -2 r^{3} R \sin (w) \cos (v)^{2}+4 r^{3} \cos (w)^{2} \cos (v)^{2} \beta+\alpha^{2} \cos (v)^{2} R^{2} \beta^{2}-4 \cos (w)^{4} \cos (v)^{2} \beta^{3} r+8 \cos (w)^{2} \cos (v)^{2} \beta^{3} r \\
& +2 R^{2} \cos (v)^{2} r \beta-2 r^{3} \cos (v)^{2} \beta-6 r^{3} \cos (w)^{2} \beta-4 \cos (v)^{2} \beta^{3} r+\cos (w)^{4} \cos (v)^{2} \beta^{4}-2 \cos (w)^{2} \cos (v)^{2} \beta^{4} \\
& -R^{2} \cos (v)^{2} \beta^{2}-\cos (w)^{2} \cos (v)^{2} R^{2} \beta^{2} \alpha^{2}-2 \alpha^{2} \cos (v)^{2} R^{2} r \beta-2 R \sin (w) \cos (v)^{2} \alpha \beta^{3}-2 R \sin (w) \cos (v)^{2} r \beta^{2} \\
& -r^{4}-6 r \alpha \beta^{2} R \sin (w)-r^{2} R^{2} \cos (v)^{2}+r^{2} \alpha^{2} \cos (v)^{2} R^{2}+4 \cos (v)^{2} r^{2} \cos (w)^{2} R \sin (w) \alpha \beta-2 r^{3} \sin (w) R \alpha \\
& +5 r^{2} \cos (w)^{4} \cos (v)^{2} \beta^{2}-10 r^{2} \cos (w)^{2} \cos (v)^{2} \beta^{2}+5 r^{2} \cos (v)^{2} \beta^{2}+11 r^{2} \cos (w)^{2} \beta^{2}+2 r^{3} R \sin (w) \cos (v)^{2} \alpha \\
& +4 r^{2} R \sin (w) \cos (v)^{2} \beta+6 r^{2} R \sin (w) \alpha \beta+4 r \cos (w)^{4} \beta^{3}-6 r^{2} \beta^{2}+4 r^{3} \beta-2 R \cos (w)^{2} \sin (w) \beta^{3} \alpha \\
& \left.\left.-5 r^{2} \cos (w)^{4} \beta^{2}-4 r^{2} R \cos (w)^{2} \sin (w) \alpha \beta+\alpha^{2} R^{2} \beta^{2} \cos (w)^{2}-\cos (w)^{4} \beta^{4}+2 \cos (w)^{2} \beta^{4}\right)\right] \\
& \times 1 /\left[\left(r(r-\beta) \alpha\left(R^{2} \alpha+r^{2}-r \beta+r \sin (w) R-R \sin (w) \beta+R \sin (w) r \alpha-r^{2} \cos (w)^{2}+r \cos (w)^{2} \beta\right)\right)\right],
\end{aligned}
$$

$$
\begin{aligned}
T_{23}= & \sin (v) \cos (v)\left[2 \alpha^{2} R^{2} r \beta \cos (w)^{2}-6 \cos (w)^{2} \beta^{2} R \sin (w) r \alpha-2 r^{3} \cos (w)^{4} \beta-2 R \sin (w) \alpha \beta^{3}\right. \\
& +\beta^{4}-4 \beta^{3} r+\alpha^{2} R^{2} \beta^{2}-2 R^{2} \beta \alpha^{2} r+8 \cos (w)^{2} \beta^{3} r+r^{2} \alpha^{2} R^{2}+2 R^{2} r \beta-2 r^{3} \sin (w) R \\
& +4 r^{3} \cos (w)^{2} \beta+6 r \alpha \beta^{2} R \sin (w)-r^{2} R^{2}+2 r^{3} \sin (w) R \alpha+4 r^{2} \sin (w) R \beta-10 r^{2} \cos (w)^{2} \beta^{2} \\
& -6 r^{2} R \sin (w) \alpha \beta-4 r \cos (w)^{4} \beta^{3}+5 r^{2} \beta^{2}-2 r R \sin (w) \beta^{2}-R^{2} \beta^{2}-2 r^{3} \beta+2 R \cos (w)^{2} \sin (w) \beta^{3} \alpha \\
& \left.+5 r^{2} \cos (w)^{4} \beta^{2}+4 r^{2} R \cos (w)^{2} \sin (w) \alpha \beta-\alpha^{2} R^{2} \beta^{2} \cos (w)^{2}+\cos (w)^{4} \beta^{4}-2 \cos (w)^{2} \beta^{4}\right] \\
& \times\left[1 /\left(r(r-\beta) \alpha\left(R^{2} \alpha+r^{2}-r \beta+r \sin (w) R-R \sin (w) \beta+R \sin (w) r \alpha-r^{2} \cos (w)^{2}+r \cos (w)^{2} \beta\right)\right)\right],
\end{aligned}
$$

$$
\begin{aligned}
T_{33}= & {\left[\left(2 \cos (v)^{2} \cos (w)^{2} R \sin (w) \alpha \beta^{3}+2 \cos (w)^{2} \cos (v)^{2} R^{2} \beta \alpha^{2} r-6 R \sin (w) \cos (v)^{2} \alpha r^{2} \beta\right.\right.} \\
& +6 R \sin (w) \cos (v)^{2} \alpha r \beta^{2}+\cos (v)^{2} \beta^{4}-r^{4} \cos (w)^{2}-6 \cos (v)^{2} \cos (w)^{2} R \sin (w) \alpha \beta^{2} r \\
& -2 r^{3} \cos (w)^{4} \cos (v)^{2} \beta-2 r^{3} R \sin (w) \cos (v)^{2}+4 r^{3} \cos (w)^{2} \cos (v)^{2} \beta+\alpha^{2} \cos (v)^{2} R^{2} \beta^{2} \\
& -4 \cos (w)^{4} \cos (v)^{2} \beta^{3} r+8 \cos (w)^{2} \cos (v)^{2} \beta^{3} r+2 R^{2} \cos (v)^{2} r \beta-2 R^{2} r \beta+2 r^{3} \sin (w) R \\
& -2 r^{3} \cos (v)^{2} \beta+2 r^{3} \cos (w)^{2} \beta-4 \cos (v)^{2} \beta^{3} r+\cos (w)^{4} \cos (v)^{2} \beta^{4}-2 \cos (w)^{2} \cos (v)^{2} \beta^{4} \\
& -R^{2} \cos (v)^{2} \beta^{2}-\cos (w)^{2} \cos (v)^{2} R^{2} \beta^{2} \alpha^{2}-2 \alpha^{2} \cos (v)^{2} R^{2} r \beta-2 R \sin (w) \cos (v)^{2} \alpha \beta^{3} \\
& -2 R \sin (w) \cos (v)^{2} r \beta^{2}+r^{4}-r^{2} R^{2} \cos (v)^{2}+r^{2} R^{2}+r^{2} \alpha^{2} \cos (v)^{2} R^{2} \\
& +4 \cos (v)^{2} r^{2} \cos (w)^{2} R \sin (w) \alpha \beta-4 r^{2} \sin (w) R \beta+5 r^{2} \cos (w)^{4} \cos (v)^{2} \beta^{2} \\
& -10 r^{2} \cos (w)^{2} \cos (v)^{2} \beta^{2}+5 r^{2} \cos (v)^{2} \beta^{2}-r^{2} \cos (w)^{2} \beta^{2}+2 r^{3} R \sin (w) \cos (v)^{2} \alpha \\
& \left.\left.+4 r^{2} R \sin (w) \cos (v)^{2} \beta+r^{2} \beta^{2}+2 r R \sin (w) \beta^{2}+R^{2} \beta^{2}-2 r^{3} \beta\right)\right] \\
& \times 1 /\left[\left(r(r-\beta) \alpha\left(R^{2} \alpha+r^{2}-r \beta+r \sin (w) R-R \sin (w) \beta+R \sin (w) r \alpha-r^{2} \cos (w)^{2}+r \cos (w)^{2} \beta\right)\right)\right],
\end{aligned}
$$

where $R$ is the major radius and

$$
\alpha=b-a, \quad \beta=a .
$$

[1] J. B. Pendry, D. Schurig, and D. R. Smith, Science 312, 1780 (2006).

[2] U. Leonhardt, Science 312, 1777 (2006).

[3] A. Greenleaf, Y. Kurylev, M. Lassas, and G. Uhlmann, Commun. Math. Phys. 275, 749 (2007).
[4] R. V. Kohn, H. Shen, M. S. Vogelius, and M. I. Weinstein, Inverse Probl. 24, 015016 (2008).

[5] D. Schurig, J. J. Mock, B. J. Justice, S. A. Cummer, J. B. Pendry, A. F. Starr, and D. R. Smith, Science 314, 977 (2006). 
[6] W. X. Jiang, T. J. Cui, X. M. Yang, Q. Cheng, R. Liu, and D. R. Smith, Appl. Phys. Lett. 93, 194102 (2008).

[7] A. Greenleaf, Y. Kurylev, M. Lassas, and G. Uhlmann, Phys. Rev. Lett. 99, 183901 (2007).

[8] U. Leonhardt and T. Tyc, Science 323, 110 (2009).

[9] J. Li and J. B. Pendry, Phys. Rev. Lett. 101, 203901 (2008).

[10] R. Liu, C. Ji, J. J. Mock, J. Y. Chin, T. J. Cui, and D. R. Smith, Science 323, 366 (2008).

[11] J. Valentine, J. Li, T. Zentgraf, G. Bartal, and X. Zhang, Nat. Mater. 8, 569 (2010).

[12] L. H. Gabrielli, J. Cardenas, C. B. Poitras, and M. Lipson, Nat. Photonics 3, 461 (2009).

[13] I. I. Smolyaninov, New J. Phys. 10, 115033 (2008).

[14] P. A. Huidobro, M. L. Nesterov, L. Martin-Moreno, and F. J. Garca-Vidal, Nano Lett. 10, 1985 (2010).

[15] M. Bashevoy, V. Fedotov, and N. Zheludev, Opt. Express 13, 8372 (2005).

[16] Y. Liu, T. Zentgraf, G. Bartal, and X. Zhang, Nano Lett. 10, 1991 (2010).

[17] M. Kadic, S. Guenneau, and S. Enoch, Opt. Express 18, 12027 (2010).

[18] J. Renger, M. Kadic, G. Dupont, S. Acimovic, S. Guenneau, R. Quidant, and S. Enoch, Opt. Express 18, 15757 (2010).

[19] T. Zentgraf, Y. Liu, M. H. Mikkelsen, J. Valentine, and X. Zhang, Nat. Nanotech. 6, 151 (2011).

[20] M. Kadic, S. Guenneau, S. Enoch, P. A. Huidobro, L. Martín-Moreno, F. J. García-Vidal, J. Renger, and R. Quidant, Nanophotonics 1, 51 (2012).

[21] W. L. Barnes, A. Dereux, and T. W. Ebbesen, Nature (London) 424, 824 (2003).

[22] T. W. Ebbesen, H. J. Lezec, H. F. Ghaemi, T. Thio, and P. A. Woff, Nature (London) 391, 667 (1998).

[23] J. B. Pendry, L. Martin-Moreno, and F. J. Garcia-Vidal, Science 305, 847 (2004).
[24] F. J. Garcia de Abajo, G. Gomez-Santos, L. A. Blanco, A. G. Borisov, and S. V. Shabanov, Phys. Rev. Lett. 95, 067403 (2005).

[25] A. Alu and N. Engheta, Phys. Rev. E 72, 016623 (2005).

[26] N. A. Nicorovici, R. C. McPhedran, and G. W. Milton, Phys. Rev. B 49, 8479 (1994).

[27] B. Baumeier, T. A. Leskova, and A. A. Maradudin, Phys. Rev. Lett. 103, 246803 (2009).

[28] Q. Cheng, T. J. Cui, W. X. Jiang, and B. G. Cai, New J. Phys. 12, 063006 (2010).

[29] E. E. Narimanov and A. V. Kildishev, Appl. Phys. Lett. 95, 041106 (2009).

[30] D. A. Genov, S. Zhang, and X. Zhang, Nat. Phys. 5, 687 (2009).

[31] M. Kadic, G. Dupont, S. Guenneau, and S. Enoch, arXiv:1102.2372v1.

[32] Y. Huang, Y. Feng, and T. Jiang, Opt. Express 15, 11133 (2007).

[33] F. Magnus, B. Wood, J. Moore, K. Morrison, G. Perkins, J. Fyson, M. C. K. Wiltshire, D. Caplin, L. F. Cohen, and J. B. Pendry, Nat. Mater. 7, 295 (2008).

[34] W. Cai, U. K. Chettiar, A. V. Kildiev, and V. M. Shalaev, Nat. Phot. 1, 224 (2007).

[35] H. Whitney, Geometric Integration Theory (Princeton University Press, Princeton, 1957).

[36] A. Nicolet, J. F. Remacle, B. Meys, A. Genon, and W. Legros, J. Appl. Phys. 75, 6036 (1994).

[37] www.comsol.com.

[38] A. Greenleaf, Y. Kurylev, M. Lassas, and G. Uhlmann, Phys. Rev. Lett. 101, 220404 (2008).

[39] A. Greenleaf, Y. Kurylev, M. Lassas, and G. Uhlmann, Opt. Express 15, 12717 (2007).

[40] B. Wood and J. B. Pendry, J. Phys.: Condens. Matter 19, 076208 (2007).

[41] F. Gomory et al., Science 335, 1466 (2012).

[42] Y. You, G. W. Kattawar, and P. Yang, Opt. Express 17, 6591 (2009). 